\title{
Colored ultrathin hybrid photovoltaics with high quantum efficiency
}

\author{
Kyu-Tae Lee*, Jae Yong Lee*, Sungyong Seo and L Jay Guo
}

Most current solar panels are fabricated via complex processes using expensive semiconductor materials, and they are rigid and heavy with a dull, black appearance. As a result of their non-aesthetic appearance and weight, they are primarily installed on rooftops to minimize their negative impact on building appearance. The large surfaces and interiors of modern buildings are not efficiently utilized for potential electric power generation. Here, we introduce dual-function solar cells based on ultrathin dopant-free amorphous silicon embedded in an optical cavity that not only efficiently extract the photogenerated carriers but also display distinctive colors with the desired angle-insensitive appearances. Light-energy-harvesting colored signage is demonstrated. Furthermore, a cascaded photovoltaics scheme based on tunable spectrum splitting can be employed to increase power efficiency by absorbing a broader band of light energy. This study pioneers a new approach to architecturally compatible and decorative thin-film photovoltaics.

Light: Science \& Applications (2014) 3, e215; doi:10.1038/lsa.2014.96; published online 24 October 2014

Keywords: color filters; nanocavities; organic-inorganic nanostructures; photovoltaics; spectrum splitting

\section{INTRODUCTION}

It is a dream of many to utilize solar energy to solve the world energy crisis and to curb climate change. Every minute, the earth receives sufficient energy from the Sun to satisfy the world's total energy requirements for the entire year. Photovoltaics (PVs) are promising platforms for the harvesting of this inexhaustible solar energy, and new device concepts with improved efficiency are constantly emerging in this field. The currently widespread inorganic thin-film PVs are dull and black in color and are not visually appealing. Therefore, they are normally installed on rooftops. Thus, there is a strong need to develop multifunctional PVs that can offer attractive design features, e.g., with desired colors and patterns, to allow them to be easily integrated with both interior and exterior architectural features, such as facades, skylights, windows and offices.

The black color of the traditional PV panel is caused by the use of a thick semiconductor layer to absorb most incident light. To create colored PV panels, thinner semiconductor layers must be used. To produce colors, one may potentially exploit the concepts and designs studied in the field of structural colors. ${ }^{1-7}$ For instance, the integration of organic semiconductors into specially designed reflective color filters has previously been demonstrated to permit the generation of electric power through the simultaneous harvesting of the absorbed light. ${ }^{8}$ However, organic PVs are known to suffer from oxygen and moisture sensitivity and have short lifetimes. ${ }^{9}$ The nanostructures used in this previous demonstration are also difficult to scale to practical large areas. It is also desired that the color appearance should not change with viewing angle, but the previous approach cannot satisfy this requirement because it uses gratings to couple the incident light.
Clearly, a general and simpler design principle is needed to naturally integrate the semiconductor materials into the structure while addressing both the optical and electrical requirements.

The simplest approach to obtaining a dual-function colored PV device is to use a metal-semiconductor-metal structure. Because of partial reflection from the metals, a Fabry-Pérot (F-P) resonator can be formed to produce the desired reflected colors, which are determined by the thickness of the semiconductor. The two metal layers also simultaneously function as electrodes to extract the photocurrent produced by the light absorbed by the semiconductor layer. However, typical F-P resonances are known to exhibit strong angle dependence, which will need to be addressed for our application. Amorphous silicon (a-Si) is chosen because it is a mature semiconductor technology that is already used in the production of a-Si PVs and because its deposition is also compatible with the proposed metal-semiconductor-metal structure. Moreover, it can suppress the angle dependence of the resonance, as will be explained later. However, a quick calculation indicates that one would need an a-Si layer of $10-30 \mathrm{~nm}$ to produce reflected colors in the visible range. This is challenging because it is known that the doped layers alone in traditional a-Si PV already constitute a thickness of $40-50 \mathrm{~nm} .{ }^{10}$ Evidently, a new device structure is needed to comply with both the optical and electrical characteristics required of dual-function solar cells.

In this report, we present an approach to addressing these challenges by introducing an a-Si/organic hybrid solar cell structure, which allows for the use of an ultrathin undoped a-Si layer and can produce desirable reflected colors. Furthermore, the reflected colors are neither sensitive to the angle of incidence for angles of up to $60^{\circ}$ nor to the polarization state of the incident light. We borrow the underlying 
concept from organic PVs and use very thin inorganic and organic charge transport layers to sandwich the undoped intrinsic (i-) a-Si active layer at the anode and cathode interfaces. Because the thickness of the ultrathin photoactive layer ranges from $10 \mathrm{~nm}$ to $27 \mathrm{~nm}$ (an order of magnitude smaller than that of the typical $\mathrm{p}-\mathrm{i}-\mathrm{n}$ a-Si) ${ }^{11}$ and because of the elimination of the $\mathrm{n}$ - and $\mathrm{p}$ - doped regions, which are known to cause significant charge recombination, ${ }^{12}$ the measured external quantum efficiency (EQE) of the devices closely matches the calculated optical absorption of the thin a-Si layer. As a result, the colored hybrid cells can produce a power conversion efficiency close to $3 \%$, despite the use of an a-Si layer ten times thinner than that of traditional a-Si PVs. This achievement would have not been possible without the optical resonance effect and the suppression of charge recombination. By combining differently colored PV cells, arbitrary patterns or images can be obtained. Furthermore, we propose that by arranging three cells that absorb different ranges of the solar spectrum and whose reflection spectra compensate for each other, a cascaded configuration can be created that uniquely exploits the spectrum-splitting concept to harvest a broad spectrum of solar light for higher efficiency.

\section{MATERIALS AND METHODS}

The dual-function a-Si/organic hybrid solar cells are constructed on transparent fused silica substrates. The main components of the hybrid cells are an anode, an ultrathin a-Si layer, and a cathode with a thick silver (Ag) layer, as shown in Figure 1a. For the anode, a dielectric-metal structure (i.e., tungsten trioxide $\left.\left(\mathrm{WO}_{3}\right)-\mathrm{Ag}\right)$ is used to produce relatively high transmission. Following the anode, a high work function vanadium oxide $\left(\mathrm{V}_{2} \mathrm{O}_{5}\right)^{13}$ serves as a hole-transporting layer for the undoped a-Si and simultaneously blocks electrons. Following the deposition of the a-Si layer, a thin organic layer of indene- $\mathrm{C}_{60}$ bisadduct (ICBA) ${ }^{14}$ is deposited to serve as an electrontransporting layer for the a-Si because its lowest unoccupied molecular orbital is well aligned with the a-Si conduction energy band, as shown in Figure 1b. Another organic material of bis-adduct fullerene surfactant $\left(\mathrm{C}_{60} \text { surfactant }\right)^{15}$ is spin cast on top of the ICBA to lower the Ag work function, thus forming an ohmic contact between the ICBA and the thick Ag cathode. All anode and cathode materials, except the ICBA, were thermally evaporated with the same thicknesses, regardless of the device type, without breaking the vacuum below $1 \times 10^{-6} \mathrm{mbar}$. The ICBA was spin cast. The undoped a-Si layers were deposited using a plasma-enhanced chemical vapor deposition tool for colored PV devices. An anode of $\mathrm{WO}_{3} / \mathrm{Ag}$ was thermally deposited onto a fused silica substrate, producing a sheet resistance below $6 \Omega \mathrm{sq}^{-1}$. In the same chamber, without breaking the high vacuum ( $10^{-6}$ mbar $), \mathrm{V}_{2} \mathrm{O}_{5}$ was deposited at a rate of $0.2 \AA^{-1}$ to form a hole-transport layer. An undoped a-Si photoactive layer was deposited at $240{ }^{\circ} \mathrm{C}$ using $\mathrm{SiH}_{4}$ gas in a plasma-enhanced chemical vapor deposition apparatus with 13.56-MHz-radiofrequency power. To create electron-transporting
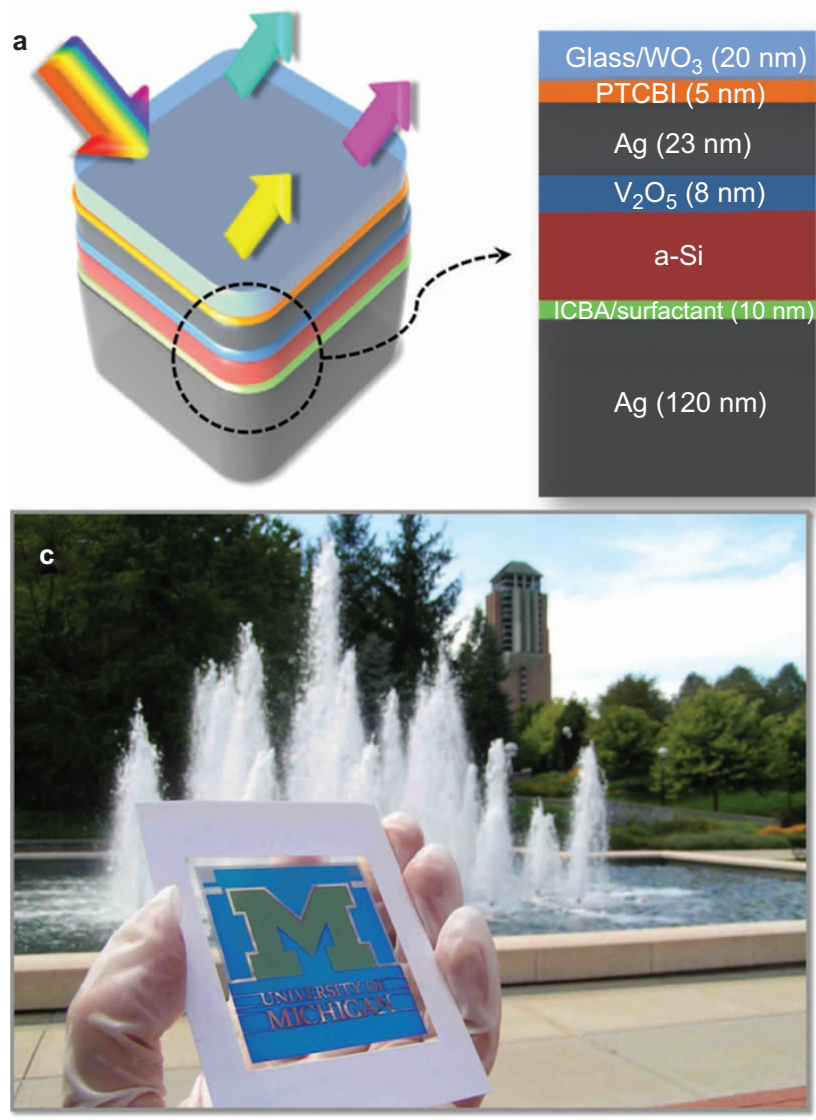
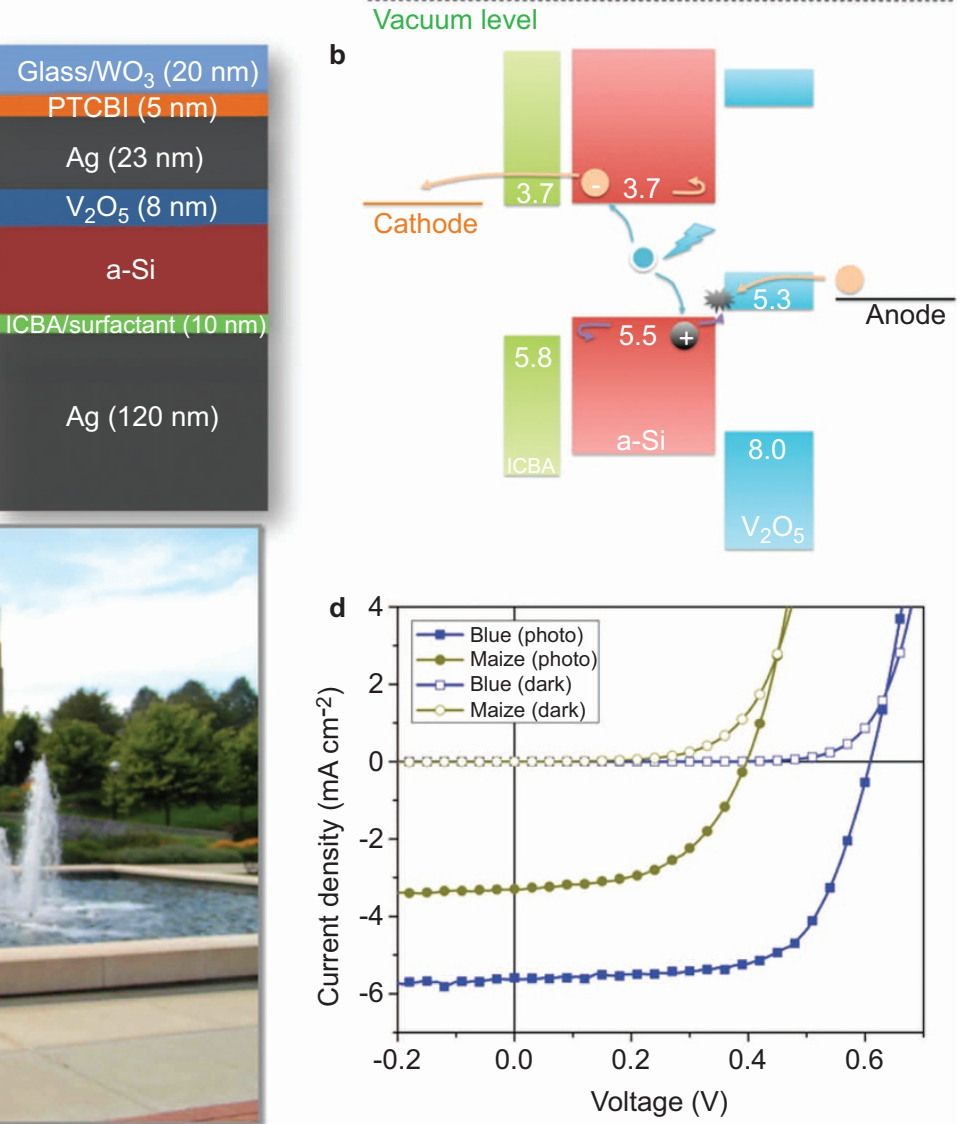

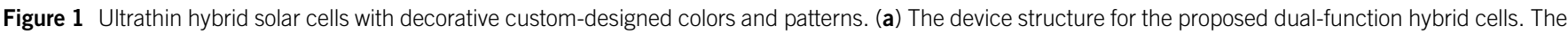

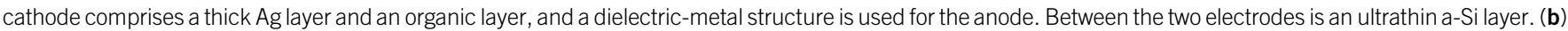

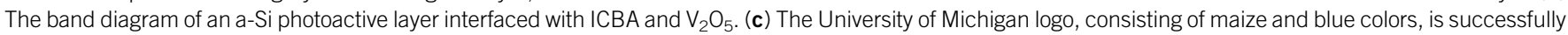

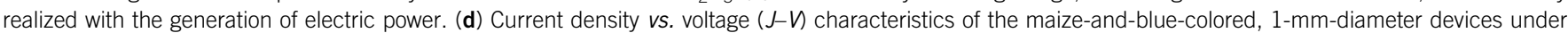
AM1.5 illumination. AM, air mass; a-Si, amorphous silicon; ICBA, indene- $\mathrm{C}_{60}$ bisadduct. 
and Ag work function tuning layers, ICBA/chlorobenzene solution and $\mathrm{C}_{60}$ surfactant were then spin cast onto the a-Si layer. Finally, thick Ag was thermally evaporated on top of the previous layers as a cathode electrode and a strong reflector. The electrical performance of our colored PVs was characterized through current density $v s$. voltage $(J-V)$ measurements. Circular-shaped devices of $1 \mathrm{~mm}$ in diameter were tested under illumination with AM1.5 (AM, i.e., air mass) simulated sunlight $\left(100 \mathrm{~mW} \mathrm{~cm}^{-2}\right)$. The intensity of the light was uniformly distributed throughout the cell area by the optical set-up. An instrument (Keithley 2400; Keithley Instruments, Cleveland, $\mathrm{OH}$, USA) was used for the data acquisition of the current and voltage values during $J-V$ characterization. The power conversion efficiency of each colored cell was determined from the average values of $J_{\mathrm{sc}}, V_{\mathrm{oc}}$ and fill factor of multiple cells on the same substrate. For optical performance characterization, the reflection spectra of the colored PV cells at normal incidence were measured using a spectrometer (HR4000CG; Ocean Optics, Dunedin, FL, USA). A halogen lamp was used as a white light source. Angular dependent spectra were obtained using a variable-angle spectroscopic ellipsometer (VASE; J. A. Woollam Co., Lincoln, NE, USA) from $15^{\circ}$ to $60^{\circ}$ for both s- and ppolarizations. Simulations of the electric field profiles, the reflection, the absorption in the a-Si layer and the angular dependent spectra were performed using a transfer matrix method. Broadband white light from $400 \mathrm{~nm}$ to $800 \mathrm{~nm}$ was used as the input illumination. The complex wavelength-dependent optical constants of all materials were measured using a spectroscopic ellipsometer (M-2000; J. A. Woollam Co.) with a B-spline fitting function and were then used in the simulations.

\section{RESULTS AND DISCUSSION}

In the anode structure, a thin Ag layer $(23 \mathrm{~nm}$ ) forms a semitransparent anode and also provides sufficient reflectivity to form the F-P resonance with the thick Ag cathode reflector. Additionally, the thickness of the Ag determines the conductivity of the anode, of which the sheet resistance is below $6 \Omega \mathrm{sq}^{-1}$. A wetting layer, perylenetetracarboxylic bis-benzimidazole (PTCBI), ${ }^{16}$ is utilized for the formation of a continuous Ag film, which can reduce the scattering loss significantly. Although the band gap of PTCBI is $2.2 \mathrm{eV}$, it is found that the absorption loss of a few-nanometer PTCBI layer is trivial. We should note that the behavior is quite different if the PTCBI is replaced by Ge, a commonly used seed layer for thin Ag films, ${ }^{17}$ as Ge causes much more significant absorption in the visible range. A detailed discussion is presented in the Supplementary Information.

As a demonstration (Figure 1c), we fabricated an electric-powergenerating University of Michigan logo ( 3 inches $\times 2.3$ inches). The vivid maize and blue colors are evident in the image. The $J-V$ performances of the 1-mm-diameter devices are depicted in Figure 1d. In principle, any colored image can be designed using shadow masks and colored PVs.

In a typical F-P resonator based on a transparent medium, the resonance wavelength blue-shifts when the incidence angle increases because of the increase in the propagation phase in the dielectric medium. This angle-dependent color appearance is not desirable for colored PV panels. However, our PV cells display strong, angle-independent colors. Photographs of the fabricated colored PVs at various angles of incidence $\left(15^{\circ}, 30^{\circ}\right.$ and $\left.60^{\circ}\right)$ are provided in Figure $2 \mathrm{a}$, and the cyan, magenta and yellow (CMY) colors exhibit high contrast and do not change their color appearance even at high viewing angles. The reason for the strong, angle-independent resonance that is observed is as follows. For a highly absorbing material (e.g., a-Si, for which the imaginary part of the refractive index is comparable to the real part at visible frequencies), the phase change upon the reflection at the interface between an optically lossy medium and a metal varies from 0 to $\pi$ because of the complex refractive index of the metal in the visible range and the large absorption of the lossy medium, which is markedly different from the case of a transparent medium. These non-trivial reflection phase changes enable the desired resonance to be created in an ultrathin cavity where the thickness of the absorbing material is much smaller than the wavelength of the incident light. ${ }^{18-20}$ Our PV cell exploits this property to produce an angle-insensitive color appearance because the propagation phase through the ultrathin Si layer is reduced and can be partially cancelled out by the reflection phase shifts at the interface between the a-Si and the metal. ${ }^{21,22} \mathrm{~A}$ numerical analysis of the reflected spectra at various angles was performed using a transfer matrix method, and the results are presented in Figure $2 \mathrm{~b}-2 \mathrm{~d}$. The resonance (reflection dip) of each color is invariant with respect to the angle of incidence for angles of up to $60^{\circ}$, as indicated by the flat dispersion curve. Experimentally, these angular behaviors were measured and confirmed using a variable-angle spectroscopic ellipsometer (J. A. Woollam Co.) for angles of $15^{\circ}-60^{\circ}$ under p-polarized light illumination, as illustrated in Figure $2 \mathrm{e}-2 \mathrm{~g}$. The measured reflected responses at the various angles agree well with the results of the simulation. In addition, the light absorption is strongly confined within the thin a-Si layer at the resonance wavelength (Figure $2 \mathrm{~h}-2 \mathrm{j}$ ), facilitated by the higher $n$ and $k$ values of a-Si compared with the other layers. The high concentration of the field within the a-Si film contributes to the photocurrent generation (Supplementary Eq. S1).

Another key feature of our designed colored cells is their low sensitivity to the polarization state of the incident light, which means that light of any polarization can be fully absorbed by the a-Si active layer and thus, contribute to the photocurrent generation, which is a highly desirable feature, as sunlight and ambient light are mostly unpolarized. In the Supplementary Information, the angular dependence for s-polarized light is also presented.

Next, we discuss the benefit of the hybrid cell structure without pand $\mathrm{n}$ - doped layers in the a-Si cell. The thin organic and metal-oxide interfacial layers have much lower refractive indices than do doped a-Si layers and therefore do not significantly affect the optical resonance. Moreover, they also facilitate the extraction of the photogenerated charge to the electrodes with significantly reduced recombination. In Figure $3 \mathrm{a}$, the simulated absorption spectra of the a-Si active layers for the CMY colored cells are compared with the measured EQE values for these devices. On the whole, the two spectra match remarkably well. This finding indicates that most of the absorbed photons are harvested and contribute to the photocurrent with negligible electron-hole recombination. This high efficiency arises because the thickness of the active layer is much smaller than the typical charge-diffusion length in $\mathrm{a}-\mathrm{Si},{ }^{23,24}$ and therefore, most photogenerated charges are efficiently extracted to the electrodes via the organic/inorganic (electron/hole) transporting layers. For the magenta and cyan devices, the EQE spectra exhibit slightly lower profiles than the simulated absorption profiles for a-Si. This discrepancy is attributed to fabrication imperfections, such as physical defects and surface roughness, which affect the resonance behavior. In particular, the cyan color has the strongest resonance because of the lower absorption by a-Si at longer wavelengths; it is evident that a scenario with stronger interference (i.e., more round trips of light reflected by the two electrodes) can be more strongly affected by defects and imperfections.

Considering the EQE characteristics of the hybrid cells presented in Figure 3a, we expect fairly similar electric-current performance from 


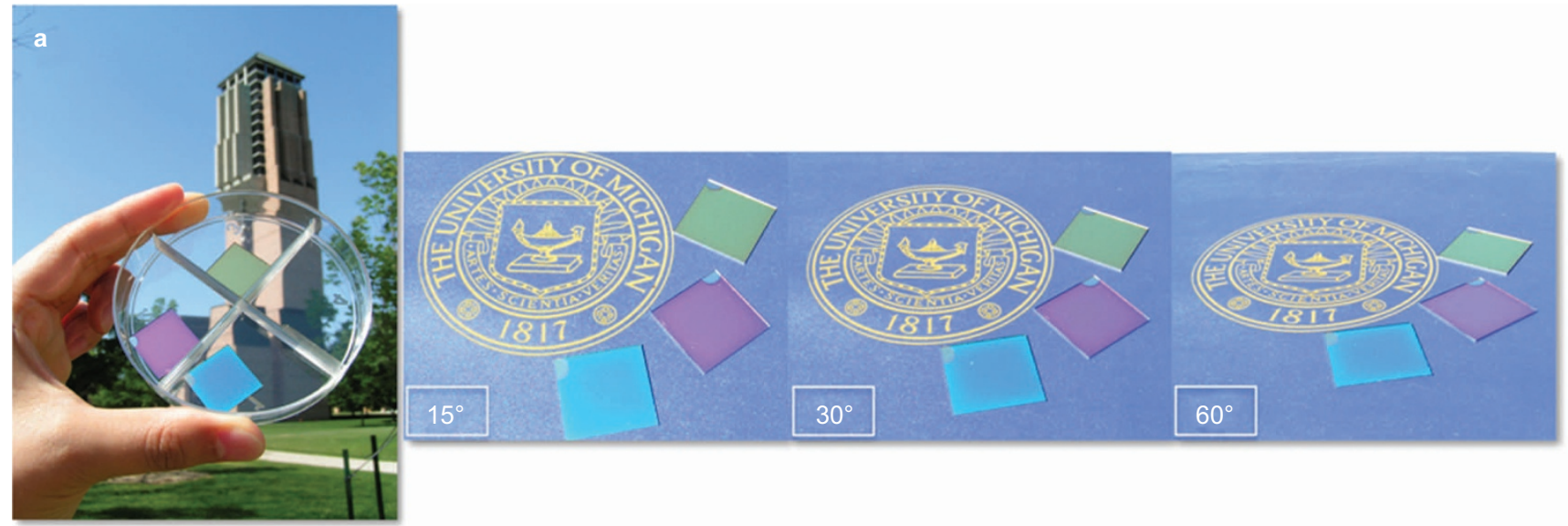

Simulation

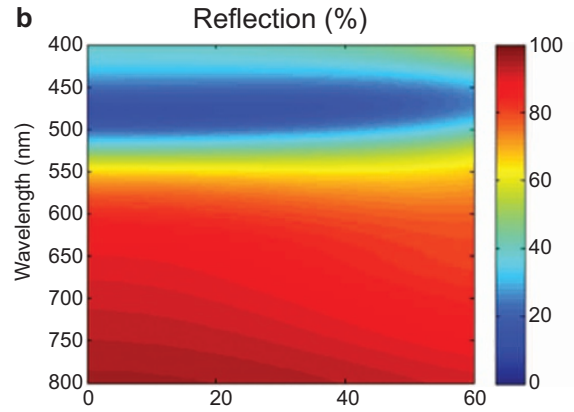

Angle of incidence $\left({ }^{\circ}\right)$

Measurement

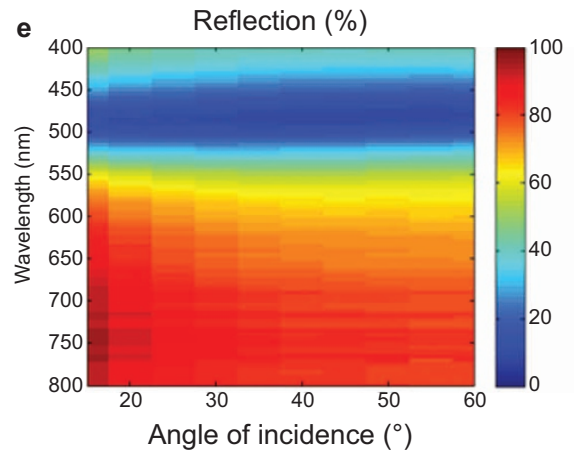

h

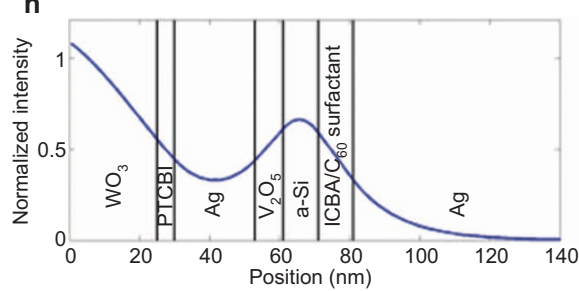

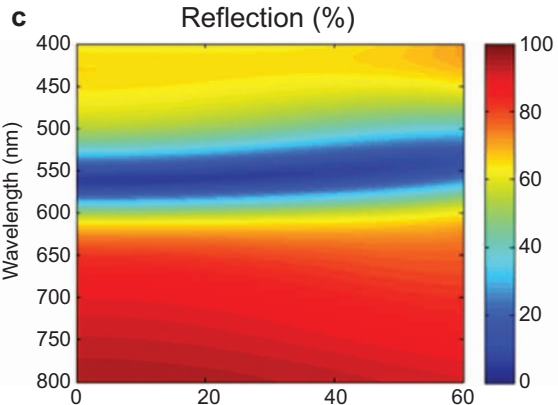

Angle of incidence $\left({ }^{\circ}\right)$
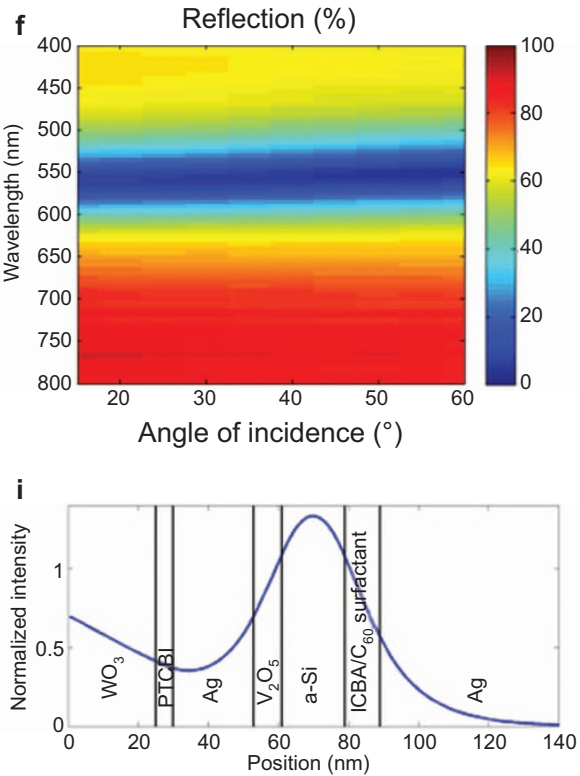

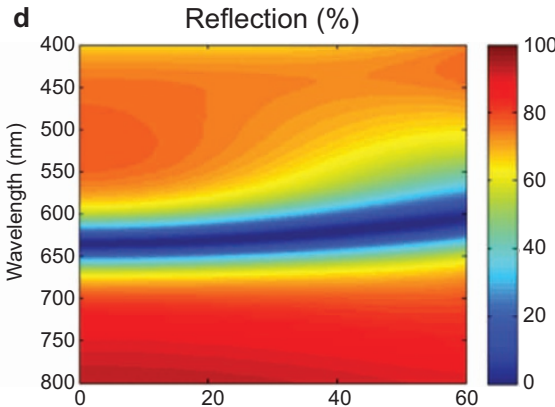

Angle of incidence $\left(^{\circ}\right)$
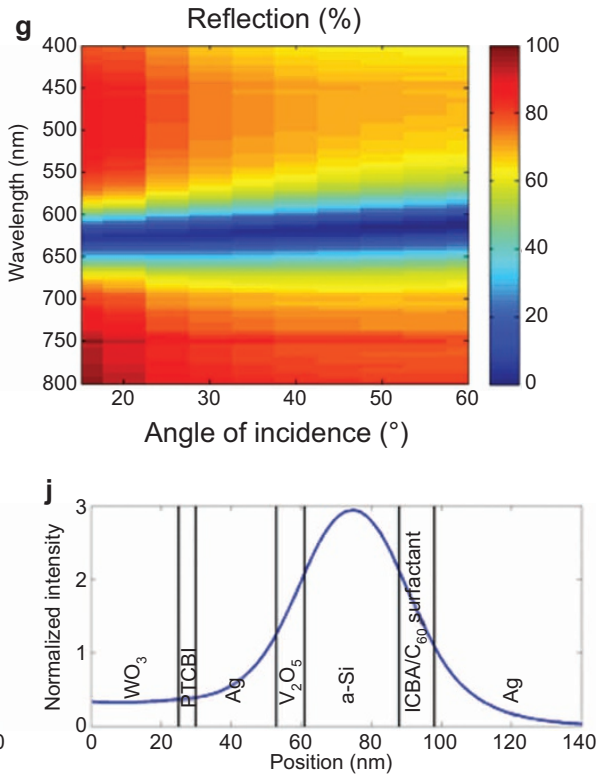

Figure 2 Incidence-angle dependence of the behavior of the reflected colors. (a) Images of the fabricated devices displaying vivid CMY colors. The colored cells clearly demonstrate angle-insensitive performance at large angles of incidence $\left(15^{\circ}, 30^{\circ}\right.$ and $60^{\circ}$ ). (b-d) Dispersion curves (for p-polarized light) obtained through simulation (transfer matrix method) for yellow (10 nm), magenta (18 nm) and cyan ( $27 \mathrm{~nm}$ ), respectively. The flat dispersion curves indicate that the devices have low sensitivity to angles of incidence ranging from $0^{\circ}$ to $60^{\circ}$. The color maps represent the intensity values for the reflected light. (e-g) Experimental dispersion curves measured using an ellipsometer and corresponding to those in $\mathbf{b}-\mathbf{d}$. $(\mathbf{h}-\mathbf{j})$ The normalized intensity distribution of the electric field inside the a-Si layer at the resonance for each individual color corresponding to $\mathbf{b}-\mathbf{d}$. a-Si, amorphous silicon; CMY, cyan, magenta and yellow; ICBA, indene- $\mathrm{C}_{60}$ bisadduct.

the yellow cell $(10 \mathrm{~nm})$ and the magenta cell $(18 \mathrm{~nm})$, even though their thicknesses differ by nearly a factor of 2 . This expectation is supported by the electrical characterization, which is presented in Figure $3 b$.
The $J-V$ curves displayed in Figure $3 \mathrm{~b}$ represent the average efficiency performance measured for multiple devices of each color type (CMY). For all devices, the measured short-circuit currents presented in Figure $3 \mathrm{~b}$ agree with the integrated EQE results presented in 

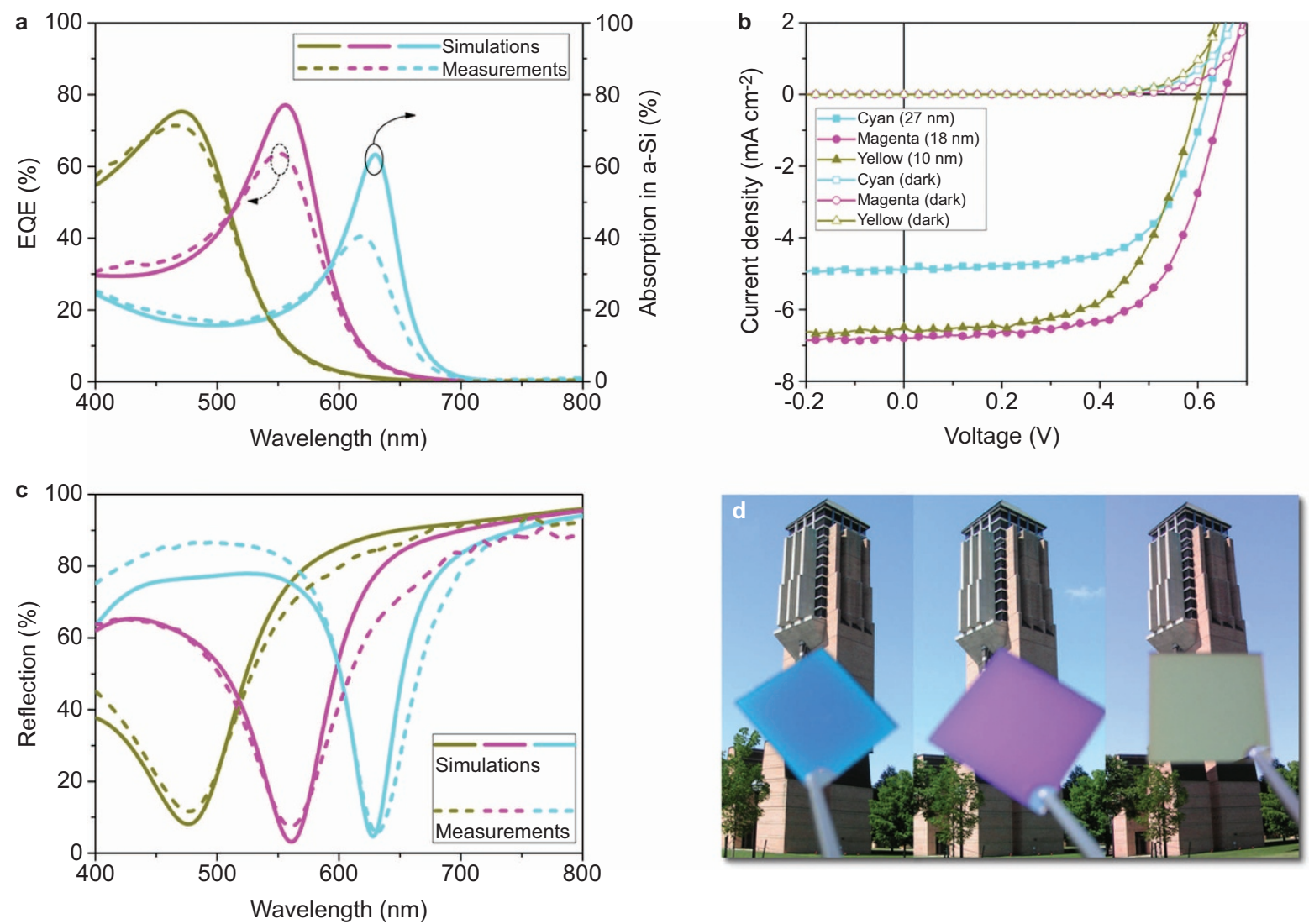

Figure 3 Optical and electrical characterizations of the fabricated dual-function devices. (a) Measured EQE spectra and calculated absorption profiles of the a-Si layers for three individual colors: CMY. (b) Measured $J-V$ characteristics of individual colored cells under both AM1.5 illumination and dark conditions. (c) Calculated (solid lines) and measured (dotted lines) reflection spectra of the CMY devices at normal incidence. The thicknesses of the a-Si layer for the CMY colors are 27,18 and $10 \mathrm{~nm}$, respectively, given fixed thicknesses of all the other layers as shown in Figure 1a. (d) A photograph of the devices reflecting CMY colors. AM, air mass; a-Si, amorphous silicon; CMY, cyan, magenta and yellow; EQE, external quantum efficiency.

Figure 3a. The yellow device has a very similar current density $\left(6.50 \mathrm{~mA} \mathrm{~cm}^{-2}\right)$ to the magenta one $\left(6.79 \mathrm{~mA} \mathrm{~cm}^{-2}\right)$, with a comparable fill factor of above $60 \%$. This result is consistent with the similar integrated EQEs seen in Figure 3a for the yellow cell $(10 \mathrm{~nm})$ and the magenta cell $(18 \mathrm{~nm})$, despite their difference in thickness. Importantly, the magenta device generates a power efficiency of nearly $3 \%$ with an undoped a-Si active layer of only $18 \mathrm{~nm}$ in thickness. This is a remarkable result when compared with the record $10 \%$ efficiency obtained for a single-junction cell using a 250-nm intrinsic a-Si layer, ${ }^{25}$ which is over 10 times thicker than our device. This result demonstrates that the efficient photon management achieved through the establishment of optical resonance and the reduced charge recombination in ultrathin a-Si plays a crucial role in our dual-function cells. More detailed information concerning the power efficiencies of the three types of devices is summarized in the Supplementary Information. Notably, all three types of devices, regardless of their thickness (10-27 nm), operated well, with fill factors over $60 \%$.

Now, we discuss the optical design principle. In the ultrathin hybrid structures described above, because of the strong F-P resonance formed between the reflecting electrodes, the device produces high absorption of a certain wavelength component while reflecting the complementary spectrum. By simply varying the thickness of the a-Si layer inside the F-P resonator, different colors can be obtained. For example, to create the CMY colors, a-Si layers of 27, 18 and $10 \mathrm{~nm}$ in thickness were used, respectively, and the corresponding resonances (reflection dips) are observed at 630, 560 and $480 \mathrm{~nm}$. Figure $3 \mathrm{c}$ presents the measured and simulated reflection spectra at normal incidence, which exhibit excellent agreement. It is worth noting that the imaginary part of a-Si becomes insignificant at longer wavelengths, which results in lessened propagation loss through the a-Si film and a correspondingly narrower bandwidth. Therefore, the cyan-colored device has the sharpest resonance among the three colors represented in Figure 3c. The photographs displayed in Figure 3d show the CMY colors reflected by our devices against a background of a tower on the university campus. The devices for the evaluation of both optical and electrical performance were all fabricated using the same process. In addition, we investigated the effects of the thickness of the top metal layer in the DMD (digital micro-mirror device) anode on the electrical performance of our hybrid cells, particularly the photocurrent $J_{\mathrm{sc}}$, and the results are summarized in the Supporting Information. In general, a thicker metal layer leads to higher reflection and therefore increases the qualityfactor ( $Q$-factor) of the F-P resonance, leading to the production of higher color purity. However, a tradeoff arises in reduced a-Si absorption and lower power conversion efficiency. Therefore, an ultrathin metal with low loss is desirable for this application, such as Ag with the introduction of a small amount of $\mathrm{Al}$ to produce a sub-10-nm smooth $\mathrm{Ag}$ film. ${ }^{26}$

Finally, we suggest another innovative use of our colored reflectiontype PVs. Recently, increasing interest has arisen in the use of the spectrum-splitting approach to spread the solar spectrum into multiple bands and use semiconductor PVs with the appropriate band gap 
for each band. This approach has the potential to yield record high efficiency without the need of engineering sophisticated vertical tandem cell structures for broadband absorption. However, spectrumsplitting approaches always require a specifically designed external element to spread the spectrum, such as a filter, ${ }^{27}$ grating, ${ }^{28}$ prism $^{29}$ or beam splitter. ${ }^{30}$ Such an external element is not required for our proposed cascaded configuration, as shown in Figure 4a: because of its strong resonance behavior, our colored PV device can absorb a certain spectral band of solar light and naturally reflect its complementary spectrum, which can be harvested by another cell with a different resonance wavelength. Simply altering the thickness of the a-Si layer in the PV cell enables the resonance to be shifted in a controllable manner. The angle-insensitive behavior of these devices is also advantageous for cascading multiple stages of PV cells. As a demonstration, we constructed a cascaded solar cell platform based on spectrum splitting, as illustrated in Figure 4a, to span a wide range of wavelengths. We designed and fabricated three cells with different thicknesses of a-Si such that they exhibit different resonances. The light is incident at an oblique angle on the first cell, which absorbs the green band and hence reflects its complementary colors. The longer wavelengths in the unabsorbed spectrum are re-utilized by the second cell, whose resonance is at $630 \mathrm{~nm}$. Finally, the third cell harvests the remaining photons in the blue region. Note that the color of each cell in Figure 4a represents its primary absorption region. The accumulated absorption spectra are represented by dotted curves.

To study the optical and electrical characteristics of the cascaded system, we calculated the absorption spectra in the a-Si and measured the EQEs of the three cells, which are compared in Figure 4b. In general, the calculated spectra are quite consistent with the experimental EQE data, with some discrepancy at longer wavelengths, as explained above (see the discussion related to Figure 3a). From the overall EQE and a-Si absorption spectra depicted in Figure 4c (sum of three profiles), it is evident that a broad range of incident light from 400 to $700 \mathrm{~nm}$ can be harvested to generate electric power, thereby validating the cascaded-cell concept. We also investigated the $J-V$ characteristics of the individual cells (Figure $4 \mathrm{~d}$ ) and the cascaded system (Figure $4 \mathrm{e}$ ), the latter of which clearly demonstrate that the short-circuit current densities of the second and third cells become further reduced as each consecutive cell receives a lesser amount of light for a-Si absorption compared with the first cell. We should note that even longer wavelength ranges can be harvested by employing lower band gap semiconductor materials in subsequent cells.

\section{CONCLUSIONS}

In summary, we have demonstrated a dual-function device that can create angle-insensitive (up to $60^{\circ}$ ) and polarization-independent
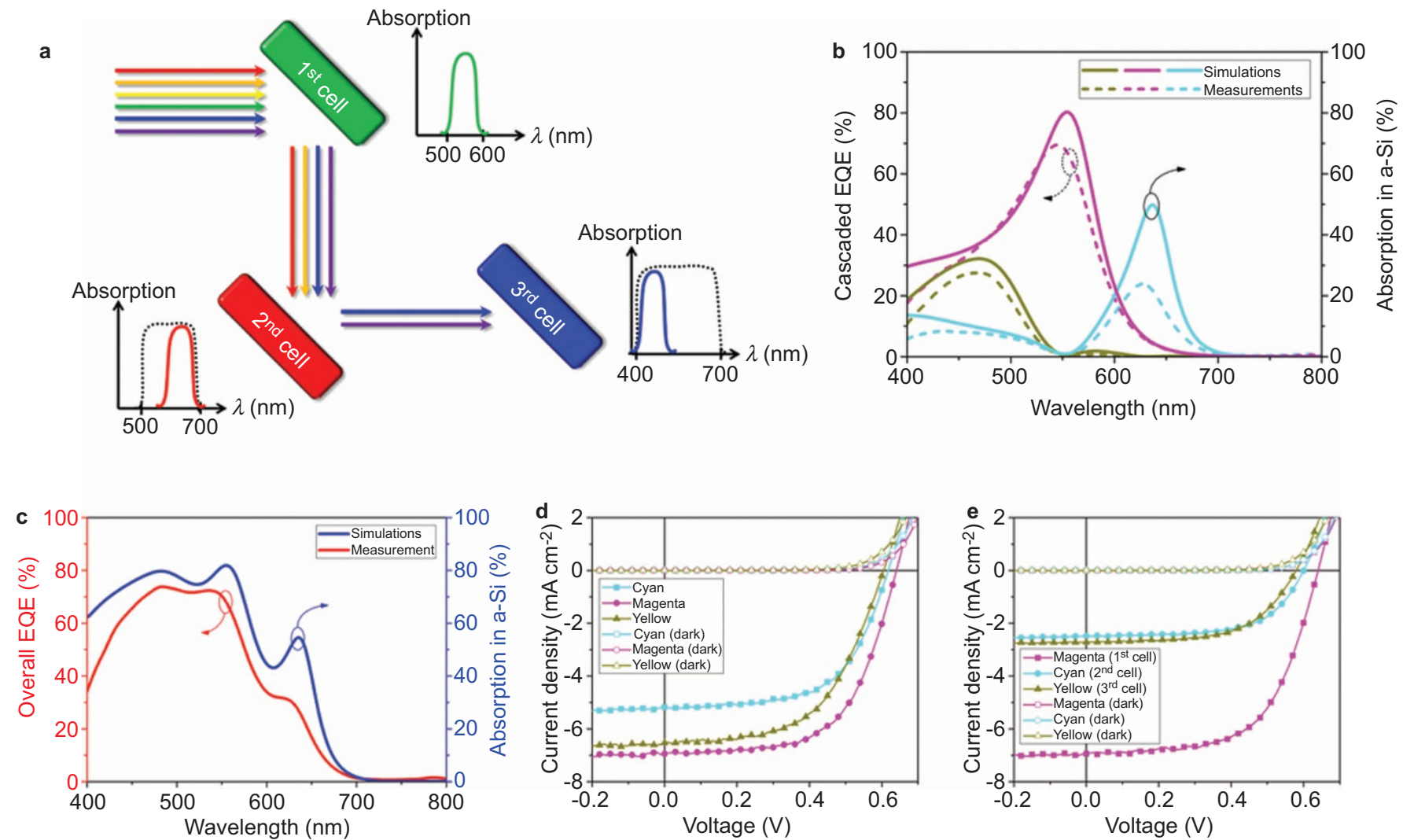

Figure 4 The achievement of photon recycling by means of spectrum splitting and cascaded light absorption. (a) A schematic illustration of the arrangement of each cell. The light is reflected by the first cell, which predominantly absorbs the green portion of the spectrum (thus reflecting a magenta color that encompasses the blue and red spectral regions), and passes into the second cell, which has a resonance that corresponds to an absorption peak in the red region and harvests longerwavelength components. By adding a third cell that reflects with a yellow color (has an absorption peak in the blue region), the remaining incident light, which still contains a strong blue spectral component, can be recycled. (b) After the application of the cascaded platform, the experimentally achieved EQE data and the corresponding numerical absorption spectra in ultrathin a-Si layer are illustrated. (c) The overall EQE profile obtained by summing the three individual EQE spectra and, for comparison, the corresponding simulated result for the total absorption in the three a-Si layers. (d) $J-V$ characteristics of the individual CMY cells under AM1.5 illumination. (e) $J-V$ performance of the three cells as measured in the cascaded platform, depicting photon recycling for the generation of electric current in consecutive cells. AM, air mass; a-Si, amorphous silicon; CMY, cyan, magenta and yellow; EQE, external quantum efficiency. 
reflected colors with high color purity. Compared with many nanostructure-based colors, which require patterned patches or subwavelength gratings, F-P resonators are much simpler to produce and scalable to industry-level production, as their fabrication involves only film-deposition processes. Our ultrathin a-Si/organic hybrid cells can generate electricity with a power conversion efficiency of up to $3 \%$. With optimization, higher efficiency should be possible. Most photons absorbed in the ultrathin undoped a-Si film can contribute to the collected photocurrent because of the suppression of electron-hole recombination, which can significantly mitigate the light-induced degradation of a-Si solar cells. ${ }^{31-33}$ It is well known that the charge recombination in $\mathrm{p}$ - and $\mathrm{n}$-doped regions is one of the major sources of charge loss in traditional $\mathrm{p}-\mathrm{i}-\mathrm{n}$ a-Si solar cells. ${ }^{12,34,35}$ Furthermore, a cascaded approach exploiting these angle-invariant resonances enables the efficient harvesting of a wide range of incident light energy. Such a scheme may possibly be implemented in combination with building-integrated PVs, e.g., by arranging building walls with desired and complementary colors such that the reflected light can also be utilized by neighboring buildings. Note that the concepts discussed in this work can be expanded to other ultrathin inorganic solar cells based on different material systems. This novel approach may have a broad influence on technologies ranging from reflective-display/lightemitting systems to decorative walls for commercial buildings, residential houses and interior furnishings.

\section{ACKNOWLEDGEMENTS}

This work was supported in part by the National Science Foundation (ECCS), Grant No. 1202046. The study of the interfacial charge-transport layers was supported by the Center for Solar and Thermal Energy Conversion, an Energy Frontier Research Center funded by the US Department of Energy, Office of Science, Basic Energy Sciences, under Award DE-SC0000957. The authors are grateful to Soluxra Inc. for providing the $\mathrm{C}_{60}$ surfactant used in the experiment. The authors would also like to thank $\mathrm{K}$ Lee for fruitful discussions and assistance with the angle measurements.

1 Xu T, Shi H, Wu YK, Kaplan AF, Ok JG et al. Structural colors: from plasmonic to carbon nanostructures. Small 2011; 7: 3128-3136.

2 Diest K, Dionne JA, Spain M, Atwater HA. Tunable color filters based on metalinsulator-metal resonators. Nano Lett 2009; 9: 2579-2583.

3 Park $\mathrm{CH}$, Yoon YT, Lee SS. Polarization-independent visible wavelength filter incorporating a symmetric metal-dielectric resonant structure. Opt Express 2012; 20: 23769-23777

4 Xu T, Wu YK, Luo XG, Guo LJ. Plasmonic nanoresonators for high-resolution colour filtering and spectral imaging. Nat Commun 2010; 1: 59.

5 Kumar K, Duan H, Hegde RS, Koh SC, Wei JN et al. Printing colour at the optical diffraction limit. Nat Nanotechnol 2012; 7: 557-561.

6 Do YS, Park JH, Hwang BY, Lee SM, Ju BK et al. Plasmonic color filter and its fabrication for large-area applications. Adv Opt Mater 2013; 1: 133-138.

7 Kaplan AF, Xu T, Guo LJ. High efficiency resonance-based spectrum filters with tunable transmission bandwidth fabricated using nanoimprint lithography. App Phys Lett 2011; 99: 143111.

8 Park HJ, Xu T, Lee JY, Ledbetter A, Guo LJ. Photonic color filters integrated with organic solar cells for energy harvesting. ACS Nano 2011; 5: 7055-7060.

9 Grossiord N, Kroon JM, Andriessen R, Blom PW. Degradation mechanisms in organic photovoltaic devices. Org Electron 2012; 13: 432-456.

10 Myong SY, Kim SS, Lim KS. Improvement of pin-type amorphous silicon solar cell performance by employing double silicon-carbide $p$-layer structure. J App/ Phys 2004, 95: $1525-1530$.
11 Söderström T, Haug FJ, Niquille X, Ballif C. TCOs for nip thin film silicon solar cells Prog Photovolt Res Appl 2009; 17: 165-176.

12 Shah AV, Schade H, Vanecek M, Meier J, Vallat-Sauvain E et al. Thin-film silicon solar cell technology. Prog Photovolt Res App/ 2004; 12: 113-142.

13 Meyer J, Zilberberg K, RiedI T, Kahn A. Electronic structure of vanadium pentoxide: an efficient hole injector for organic electronic materials. J Appl Phys 2011; 110: 033710.

14 He Y, Chen HY, Hou J, Li Y. Indene- $\mathrm{C}_{60}$ bisadduct: a new acceptor for highperformance polymer solar cells. J Am Chem Soc 2010; 132: 1377-1382.

15 O'Malley KM, Li CZ, Yip HL, Jen AK. Enhanced open-circuit voltage in high performance polymer/fullerene bulk-heterojunction solar cells by cathode modification with a $\mathrm{C}_{60}$ surfactant. Adv Energy Mater 2012; 2: 82-86.

16 Lassiter BE, Wei G, Wang S, Zimmerman JD, Diev VV et al. Organic photovoltaics incorporating electron conducting exciton blocking layers. Appl Phys Lett 2011; 98: 243307.

17 VJ L, Kobayashi NP, Islam MS, Wu W, Chaturvedi P et al. Ultrasmooth silver thin films deposited with a germanium nucleation layer. Nano Lett 2009; 9: 178-182.

18 Kats MA, Blanchard R, Genevet P, Capasso F. Nanometre optical coatings based on strong interference effects in highly absorbing media. Nat Mater 2013; 12: 20-24.

19 Kats MA, Sharma D, Lin J, Genevet P, Blanchard R et al. Ultra-thin perfect absorber employing a tunable phase change material. Appl Phys Lett 2012; 101: 221101.

20 Lee JY, Lee KT, Seo S, Guo LJ. Decorative power generating panels creating angle insensitive transmissive colors. Sci Rep 2014; 4: 4192.

21 Lee KT, Seo S, Lee JY, Guo LJ. Ultrathin metal-semiconductor-metal resonator for angle invariant visible band transmission filters. Appl Phys Lett2014; 104: 231112.

22 Lee KT, Seo S, Lee JY, Guo LJ. Strong resonance effect in a lossy medium-based optical cavity for angle robust spectrum filters. Adv Mater; e-pub ahead of print 28 July 2014; doi: 10.1002/adma.201402117

23 Goerlitzer M, Beck N, Torres P, Meier J, Wyrsch N et al. Ambipolar diffusion length and photoconductivity measurements on "midgap" hydrogenated microcrystalline silicon. J Appl Phys 1996; 80: 5111-5115.

24 Schiff EA. Low-mobility solar cells: a device physics primer with application to amorphous silicon. Sol Energy Mater Sol Cells 2003; 78: 567-595.

25 Benagli S, Borrello D, Vallat-Sauvain E, Meier J, Kroll U et al. High-efficiency amorphous silicon devices on LPCVD-ZnO TCO prepared in industrial $\mathrm{KAI}^{\mathrm{TM}}{ }_{-\mathrm{M}} \mathrm{R} \& \mathrm{D}$ reactor. In: Proceedings of the 24th European Photovoltaic Solar Energy Conference; 21-25 September 2009; Hamburg, Germany, pp2293-2298.

26 Zhang C, Zhao D, Gu D, Kim H, Ling T et al. An ultrathin, smooth, and low-loss Aldoped $\mathrm{Ag}$ film and its application as a transparent electrode in organic photovoltaics. Adv Mater 2014; 26: 5696-5701.

27 Eisler CN, Kosten ED, Warmann EC, Atwater HA. Polyhedral specular reflector design for ultra-high spectrum splitting solar module efficiencies (>50\%). Proc SPIE 2013; 8821: 88210B.

28 Escarra MD, Darbe S, Warmann EC, Atwater HA. Spectrum-splitting photovoltaics: holographic spectrum splitting in eight-junction, ultra-high efficiency module. In: Proceedings of the 39th IEEE Photovoltaic Specialists Conference; 16-21 June 2013; Tampa, FL, USA. IEEE: New York, USA, 2013, pp1852-1855.

29 Stefancich M, Zayan A, Chiesa M, Rampino S, Roncati D et al. Single element spectral splitting solar concentrator for multiple cells CPV system. Opt Express 2012; 20 . 9004-9018.

30 Mitchell B, Peharz G, Siefer G, Peters M, Gandy T et al. Four-junction spectral beamsplitting photovoltaic receiver with high optical efficiency. Prog Photovolt Res Appl 2011; 19: 61-72.

31 Deng X, Schiff EA. In:Luque A, Hegedus S, editors. Handbook of Photovoltaic Science and Engineering. Chapter 12, pp505-565. Chichester: John Wiley and Sons; 2003.

32 Staebler DL, Wronski CR Reversible conductivity changes in discharge-produced amorphous Si. Appl Phys Lett 1977; 31: 292-294.

33 Branz HM. Hydrogen collision model: quantitative description of metastability in amorphous silicon. Phys Rev B 1999; 59: 5498-5512.

34 Carlson DE, Wronski CR. Amorphous silicon solar cell. Appl Phys Lett 1976; 28: 671 673.

35 Deceglie MG, Ferry VE, Alivisatos AP, Atwater HA. Design of nanostructured solar cells using coupled optical and electrical modeling. Nano Lett 2012; 12: 2894-2900.

\footnotetext{
(i) $\Theta$ This work is licensed under a Creative Commons Attribution-

NonCommercial-NoDerivs 3.0 Unported License. The images or other third party material in this article are included in the article's Creative Commons license, unless indicated otherwise in the credit line; if the material is not included under the Creative Commons license, users will need to obtain permission from the license holder to reproduce the material. To view a copy of this license, visit http://creativecommons.org/licenses/ by-nc-nd/3.0/
} 